\title{
Project Juno: Advancing Gender Equality In Physics Careers In Higher Education In The UK
}

\author{
Marcella Bona*t \\ Queen Mary University of London \\ E-mail: m. bona@qmul . ac .uk \\ Jennifer Dyer \\ Institute of Physics \\ E-mail: Jennifer.Dyereiop.org \\ Valerie Gibson \\ Cambridge University \\ E-mail: gibson@hep.phy • cam.ac.uk
}

\section{Angela Townsend}

Institute of Physics

E-mail: Angela. Townsendeiop.org

We report here a brief summary of the Project Juno initiated and developed by the Institute of Physics (IOP) in United Kingdom. The IOP has a longstanding interest in diversity issues, particularly around the participation of girls and women in physics, who are under-represented in physics education and employment. In 2003, the IOP visited physics departments and produced a dedicated report on their gender inclusiveness: "Women in University Physics Departments: a Site Visit Scheme". Building upon the best practice identified in this influential report, in 2007 the IOP established Project Juno, an award scheme that aims to promote gender equality in higher education physics departments. The Juno Principles provide a framework for specific actions to improve the participation and retention, particularly of women, in physics careers. The main aims of the scheme are to develop an equitable, open and transparent working culture in which students and staff, men and women, can all achieve their full potential; to promote open discussion of gender and other equality issues; and to encourage departments to determine priorities for action. Departments submit for the award and are assessed by an independent Panel of physicists with longstanding experience of addressing gender equality issues. There are three levels of the scheme (Supporter, Practitioner and Champion) and almost all of the physics departments in the UK and Ireland are now participating, together with Research Institutes and one company. Currently, there are 19 Supporters, 14 Practitioners and 16 Champions.

38th International Conference on High Energy Physics

3-10 August 2016

Chicago, USA

\footnotetext{
* Speaker.

${ }^{\dagger}$ Speaker presented the poster on behalf of the Institute of Physics
} 


\section{Introduction}

From 2003-2005, the Institute of Physics (IOP) ran a successful "Women in University Physics Departments Site Visit Scheme" to address the recommendations of the International+Perceptions of UK Research in Physics and Astronomy report (2000) [1] that a special focus to attract and retain women in physics was needed from the very early stages onward. The site-visit scheme involved visiting 16 physics departments in the UK and Ireland to provide a constructive and broad assessment of their gender friendliness, producing a confidential report for each department and an overall report outlining best practice for the sector [2]. As a result of the site-visit report, the IOP developed Project Juno, building on the best practice identified, to provide recognition and reward to physics departments that were making progress to address the under-representation of women at all levels in university physics.

Project Juno was launched by the IOP in 2007 and the first awards were made in 2008. The scheme was officially evaluated in 2013 [3]. Since its inception, the remit of the scheme has widened to encompass gender equality and to attract organisations outside of physics academia. In 2017, the principles will be extended to include harassment and bullying.

Project Juno is complementary to the UK Equality Challenge Unit (ECU) Athena SWAN Charter [4], originally established to recognise commitment to advancing the careers of women in science, technology, engineering, maths and medicine (STEMM) in higher education and research. The Juno Practitioner and Champion awards are reciprocal with Athena SWAN Bronze and Silver respectively, and once a physics department achieves Juno Champion, they are encouraged and supported to work towards Athena SWAN Gold [5].

One of the main avenues of support for departments that differs from the Athena SWAN framework, is the advice and guidance provided by the IOP and the Juno Assessment Panel at every step of the journey, from Supporter to Champion. Departments have the opportunity to discuss issues with the IOP diversity team and panel members, have informal visits, and they can be buddied or mentored by nearby Champion departments. Therefore, Juno, is more than an awards scheme, but acts as a peer support network for physicists wishing to address gender equality.

\section{The Five Principles of Juno}

The five principles were developed to encompass all aspects of working life in an academic department in physics:

1. A robust organisational framework to deliver equality of opportunity and reward

2. Appointment and selection processes and procedures that encourage men and women to apply for academic posts at all levels

3. Departmental structures and systems which support and encourage the career progression and promotion of all staff and enable men and women to progress and continue in their careers

4. Departmental organisation, structure, management arrangements and culture that are open, inclusive and transparent and encourage the participation of all staff

5. Flexible approaches and provisions that enable individuals to optimise their contribution to their department, institution and to SET 
More detail on the principles can be found in the guidance provided for departments, the Project Juno Code of Practice [6].

\section{The Juno Assessment Panel and the Awards}

The Juno Assessment Panel reviews the applications to the awards. There are a currently ten panel members, all academic physicists who are in Practitioner or Champion departments but one who is an industrial physicist. ${ }^{1}$

There are three levels of award:

Supporter: To start the Juno journey and become a Supporter, the head of the department writes a letter to the IOP (there is a template available [7]) endorsing the five principles and making a commitment to work towards Practitioner and then Champion.

Practitioner: The department has to demonstrate that the Juno journey is well underway and that it has met Principle 1: A robust organisational framework to deliver equality of opportunity and reward. It also has to develop a three-year action plan to progress to Champion.

Champion The department has to demonstrate that the five principles are embedded throughout the department and that it has acted on the evidence it has found. Departments are expected to build on their Practitioner evidence base by continuing to analyse datasets, reflecting on progress and achievements, assessing the impact of the work, identifying where progress has not been as quick as expected and the reasons for this and developing a four-year action plan that will deliver lasting change in the department.

\section{Achieving the awards}

In order to present sufficient evidence to be awarded Juno Practitioner, departments are expected to provide:

- A completed Juno Practitioner Good Practice checklist [8] The Juno Good Practice Checklist is a tool for departments to use in developing their applications for Practitioner status, designed to initiate honest discussion and reflection in order for departments to establish where they are in relation to the Juno principles. The different perceptions of staff may provide an ideal starting point for discussion and provide initial ideas and evidence for the department to develop its Practitioner action plan.

- A submission detailing how the department has established its organisational framework, outlining the senior management commitment and resources allocated and the qualitative and quantitative data, broken down by gender, that the department has gathered. This includes undergraduate and postgraduate student data as well as researcher and staff data on applications, interviews, and appointments. One of the most important aspects of the Juno framework is ensuring that departments reflect on their data and use it to develop their plans for action. Analysing quantitative data with small numbers can be tricky as percentages

\footnotetext{
${ }^{1}$ It is currently chaired by Professor Val Gibson, from the Cavendish Laboratory at the University of Cambridge which, at the time of writing, is holder of the only Athena SWAN Gold award in a physics department.
} 
can fluctuate dramatically and those with smaller datasets are encouraged to aggregate data across several years (say 3-5 years). With small numbers of female staff (and students, such as $\mathrm{PhD}$ students or PDRAs) it can often be difficult to preserve anonymity in data and questionnaires and in some cases it may not be possible to present data disaggregated by gender (eg where there is only one female in the department or particular group).

- A three-year Practitioner Action Plan to fill in gaps of evidence and explore areas of concern in more depth.

Departments are encouraged to have a site visit, around two years after their Practitioner award, to provide them with feedback and guidance as they progress towards Champion. The aim of the visit is to have a positive and constructive dialogue with departments to enable them to carry out the critical reflection that is crucial for the Champion award. It is designed to provide the opportunity for a two-way dialogue, receive feedback on progress and help identify priorities for action. The aim of the feedback from the visit is to provide positive indications of the work and actions needed to move towards a successful Champion application.

When preparing their submission for Champion, departments are encouraged to reflect on the feedback from the visit and on their progress so far. Their Champion submission should cover all five principles, addressing each one in turn, and should include a 4-year action plan detailing the progress they wish to make as they work towards Athena SWAN Gold. In the action plans, departments are expected to demonstrate that the load is spread, does not fall disproportionately on one person and that the time and resources that have been allocated to carry out sufficient actions to take a department to the next level are appropriate and realistic.

\section{Progress to date}

At the time of writing, the IOP has awarded Champion status sixteen departments or schools of physics and astronomy, and to fourteen Practitioners. There are currently a further 22 Supporters, including physics research institutes and organisations outside academia. Each of our current Juno Champions has a different profile and had differing circumstances to address when submitting for Juno Champion. Some Champion departments are small, and could easily demonstrate how well communication worked on an informal level across the whole department; however, these departments also had to evidence how they formalised procedures. Some Champion departments are very large and, while they had the benefit of larger and more visible numbers of women and a higher level of resources for Juno activities, they had to evidence how Juno

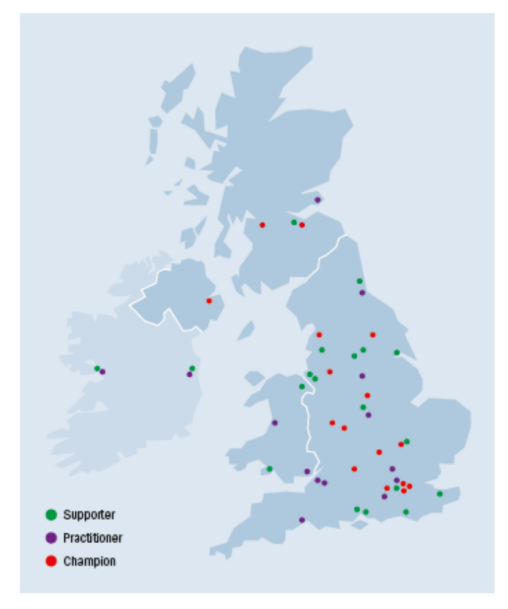

Figure 1: UK map showing geographical distributions of the 16 Juno Champions (red dots), 14 Practitioners (purple dots) and 19 Supporters (green dots). was embedded across the whole department, including in sometimes complicated group structures. Some of our Champion departments had, or still have, very low numbers of women, and had to evidence how their efforts were making a difference in a time of austerity, with recruitment freezes and little staff movement across the higher education sector. 


\section{Conclusions}

It was clear from the evaluation in 2013 that Project Juno was clearly acting as a driver for change, and concrete examples of changes in policy and practice were being articulated, with associated benefits to working environment and culture. The impact of addressing the underrepresentation of women in physics remains a long term goal. At the time of the evaluation, many staff felt that it was too early to tell whether involvement in Project Juno had had a lasting effect although some staff did report that they felt there had been increases in the visibility of women and improved working practices for all. Heads of department also reported that Juno activities had resulted in an increase in the visibility of women. There was some concern about genuine culture change taking place in departments, particularly those that had a strong group structure.

Nevertheless, the one thing that all our current Champions have in common is the determination to embed the Juno principles from the highest levels of the department down, and the most junior levels up. They have demonstrated how they engaged the whole department in their Juno work and how everyone, not just the female staff, were benefiting from both practical changes to policies and procedures and genuine departmental culture change. Our Juno Champions were not afraid to try new initiatives to decide what was going to work for them, and what they still needed to improve.

\section{References}

[1] International Perceptions of UK Research in Physics and Astronomy, Institute of Physics, May 2000. Retrieved from

https://www.iop.org/publications/iop/archive/file_52055.pdf

[2] Women in University Physics Departments: A site visit scheme 2003-2005, Institute of Physics, February 2006. Retrieved from

https://www.iop.org/publications/iop/archive/file_42616.pdf

[3] Evaluation of Project Juno: A Final Report, Institute of Physics, November 2013. Retrieved from https://www.iop.org/policy/diversity/initiatives/juno/juno-evaluation/ file_62014.pdf

[4] For more information, visit http://www.ecu.ac.uk/equality-charters/athena-swan/

[5] Journeying to the end of the rainbow? A guide for Juno Champions working towards Athena SWAN Gold, Institute of Physics, September 2016 (in print).

[6] Project Juno Code of Practice, Institute of Physics, April 2016. Retrieved from http://www.iop.org/policy/diversity/initiatives/juno/documentation/ file_42634.pdf

[7] Template letter to sign up to Project Juno: retrieved from https://www.iop.org/policy/diversity/initiatives/juno/documentation/ file_42635.pdf

[8] Juno Good Practice Checklist: retrieved from https://www.iop.org/policy/diversity/initiatives/juno/documentation/ file_42637.doc 